\title{
Selfish Caching and Facsimile Allocation over a Mobile Ad Hoc Network
}

\author{
B. Umamaheswari , P. Arivubrakan
}

\begin{abstract}
Mobile adhoc networks (MANETs) have drawn attention to multitudinous consideration because of the univerality of mobile devices as well as the developments in wireless era. MANET is a peer-to-peer multi hop cellular wireless era community which does not have both difficult and speedy infrastructure and a relevant server. Every vertex of a MANET performs like a router and communicates with every unique. There exist numerous information duplication strategies which were presented to reduce the execution squalor. All are concluded that everyone cell vertices cooperate completely from the perspective of sharing their memory vicinity. But, via a few methods few vertices might additionally behave selfishly and determine simplest to cooperate in part or never with different vertices. The selfish vertices ought to then lessen the overall information approachability within the network. From this work, we try to take a look at the influence of selfish vertices in a mobile ad hoc community in terms of reproduction issuance i.e Selfish nodes are dealt with in replica allocation.
\end{abstract}

Keywords: Energy Conservation,MANET, Selfish, Smart environment.

\section{I.INTRODUCTION}

In a mobile ad hoc community, the mobility and useful resource limitations of cellular vertices can also result in community divisioning or universal execution degradation. These cellular vertices which can be in radio variety of every different can right now talk, but remaining want intermediate vertices which help to course their packets. Mobile advert-hoc conjointly known as a wireless ad-hoc network or advert-hoc wireless community, [1]probable an ad-infinitum not the usage of a bring about sight self-configuring, infrastructure-lots less network of cell devices related wirelessly. Every device in a totally MANET is absolved to move severally in any route, and may so exchange its links to substitute devices. A huge form of MANET packages has been advanced. In MANET, every vertex behaves as a router. Those vertices in the network are chargeable to come across a course to a specific vertex and forward the facts to that vertex. As vertices inside the network are able to transfer, the infrastructure of community alternates abruptly[2]. A vertex loves to revel in the profits supplied thru manner of the belongings of numerous vertices, but it can't make its very personal beneficial resource to be had to assist remaining. This kind ofselfishness may probably cause a huge form of difficulties for a MANET. Information are greater often than no longer duplicated at vertices, except the authentic holders,

Revised Manuscript Received on 20 September, 2019.

B.Umamaheswari, Department of Computer Science and Engineering,Vel Tech Rangarajan Dr.Sagunthala R\&D Institute of Science and Technology,Chennai,India.

P.Arivubrakan, Department of Computer Science and Engineering,Vel Tech Rangarajan Dr.Sagunthala R\&D Institute of Science and Technology,Chennai,India for decorating information approachability for dealing with common community divisions. An extensive quantity of studies were these days projected for reproduction issuance in a MANET.

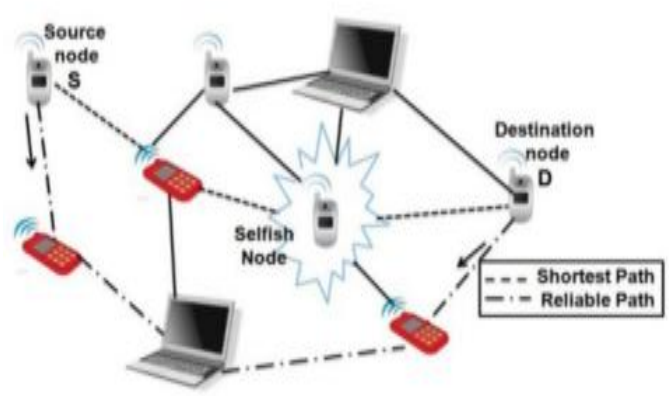

Behaviour of Selfish nodes in a Network

In practical at the identical time as managing Selfishness in MANET we want to enhance statistics approachability as well as decrease question put off, i.e question Response time, suppose the mobile vertices in a MANET altogether are having enough space region to carry every all of the duplicates as well as the proper facts. [3]The presence of selfish vertices in a community effects in a sizable reduction within the statistics approachability, whereas the get rid of duration receives extended. It can be very important to prevent the deterioration of records accessibility on the difficulty of community partition. Most essential safety hassle in MANET is cooperation of nodes. Since nodes have restricted battery electricity and which will store its battery existence, a few nodes voluntarily do not contain in forwarding method to its neighbour. This type of nodes is termed as selfish nodes and the attack associated with this is known as selfish conduct attack.

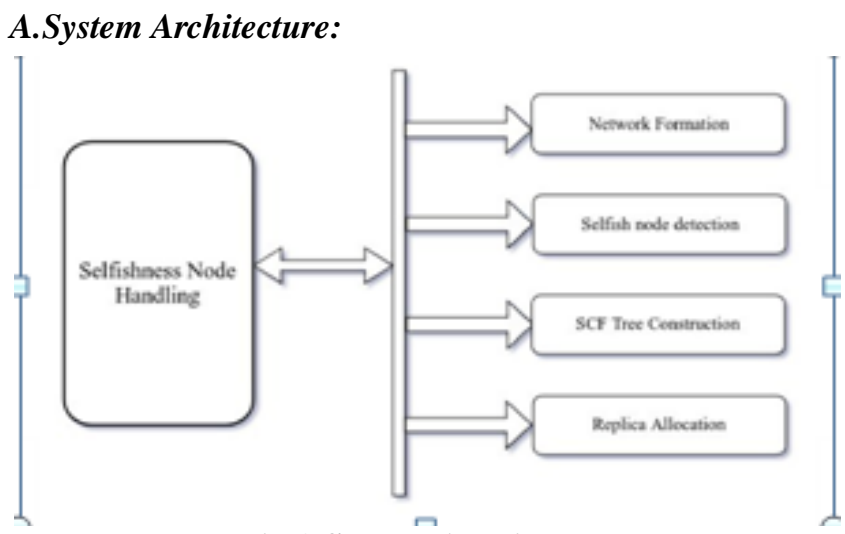

Fig.2 System Architecture

Published By: Blue Eyes Intelligence Engineering \& Sciences Publication 


\section{B.Behavior Of Selfish Nodes In Manet}

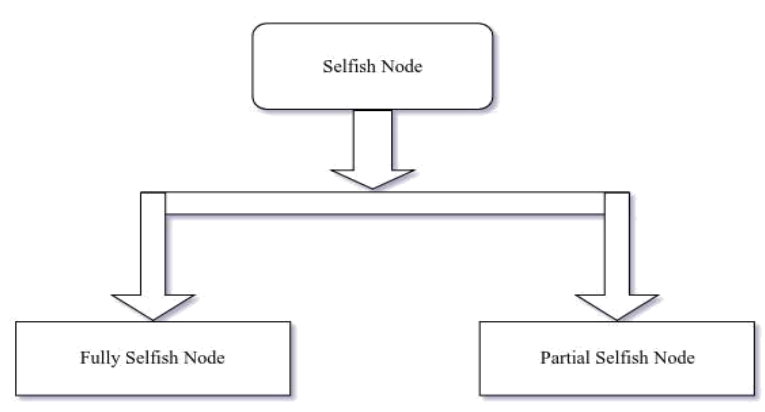

Fig3. Behaviourial diagram of selfishness nodes

Fully selfish nodes: The nodes do now not keep imitations dispensed by methods for different hubs, however apportion copies to somewhere around unique hubs for their openness.

Partial selfish nodes: The nodes utilize their memory area in segment for designated replicas through way of different nodes. These nodes apportion replicas to uncommon nodes for their availability.

Every node in a MANET has restricted memory locally and each node goes about as a data endeavour, it gives several data devices and further to a records purchaser. Every node holds realities thing replicas and keeps the replicas in neighbourhood memory space.

\section{II.EXISTING SYSTEM}

In a Network there are a few nodes, one node goes about as server and whatever remains of nodes go about as a client/customer. [4]On the off chance that a server wants to send a packet to any of the node and a node does never again acknowledge that bundle even that node has a memory space to store that bundle then such hub is called as a selfish node and drop that packets. It is fundamental to think that selfish replica allocation can be only handled by the partial selfish nodes. The three kinds nodes according to the selfish replica allocation is as follows

Type-1 node: The nodes are non-selfish nodes. The nodes hold replicas allotted through different nodes internal their memory place.

Type-2 node: All the selfish nodes come under Type- 2 node. All the allocated replicas are not maintained with the support of the distant nodes. On the other hand these replicas can get allocated to other nodes in reach of their memory space.[5]

3.Type-three node: All the partially selfish nodes come under Type-3 nodes. In this type the memory area is breached into two parts: selfish and mutual place. According to their approachability all the nodes are allocated to other nodes.

The replica allocation strategies in MANET surroundings having selfish nodes that have an impact at the general overall performance of records accessibility is as follows.

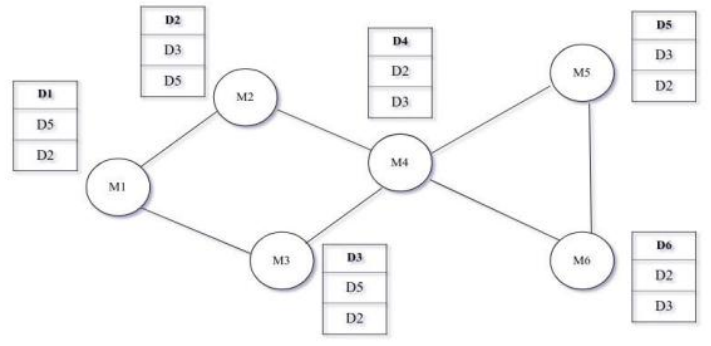

Fig 4.Node table of SAF-Method.

In SAF method, the nodes allocate replica of information consistent with the access to frequencies of that data devices. [6]In SAF method, the nodes allocate replica of data according to the get entry to frequencies of that data items. Mobile nodes with the identical get entry to frequencies to statistics objects allocate the equal replica. A mobile node can get right of entry to facts gadgets held by means of different related cellular hosts, and its miles extra possible to percentage one of a kind variety of replicas among them. The SAF approach causes low statistics accessibility at the same time as many mobile hosts have the equal access tendencies as a result a number of the data to be duplicated in hundreds of nodes. For improving data convenience of replica allocation is done by using SCF-tree.

In this, M1, M2 and so on are mobile nodes and the straight away strains among them denotes a wireless hyperlink D1, D2 and so forth are records items. The grey rectangles propose real data, at the same time as the white suggests replicas allotted.

\section{A.Dynamic Connectivity Grouping Method (DCG):}

Compared to Dynamic Access Frequency and Neighbourhood method (DAFN) the DCG method shares replicas in large businesses of mobile hosts. At every relocation period, every mobile host publicizes its host identifier.[7] After all cell hosts entire the pronounces; from the acquired owner identifier each host is aware about the associated cellular hosts and the network topology.

In every group of cellular hosts related to each wonderful, the mobile host with the bottom host identifier suffix executes an set of policies to find out bi-connected components with the network topology diagnosed by using the usage of the usage of acquired messages. Even if a mobile host belongs to more than one bi-associated thing, it is able to only belong to at least one company wherein the corresponding bi-related trouble became determined. Bby grouping cellular hosts as bi-connected additives, the enterprise organisation isn't divided no matter the truth that one cellular host disappears from the community or one link is disconnected inside the groups. 


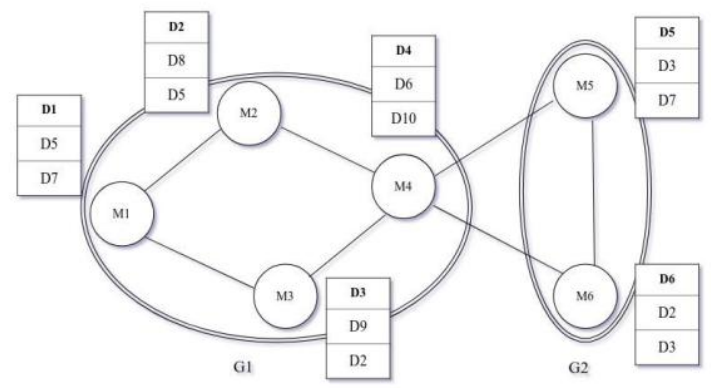

Fig 5.Node DAG Method.

As shown, DCG method two groups G1 and G2 are generated consisting of a few cellular vertices. Every mobile node broadcast its host identity and it get right of entry to frequency together with facts devices to at least one-of-a-kind nodes. By the usage of the broadcasting facts individual node analyses its bi-associated vertices. The approach to frequency of every company is computed by the usage of such as the get entry to frequencies of all the mobile nodes in that business enterprise. [8]Bestow the overall get proper of get approach to frequency of the organization, replicas of information devices have been issued until reminiscence of every cell vertex inside the number of individuals turns into exhaustive.

\section{B.Dynamic Access Frequency and Neighbourhood method $(\mathrm{DAFN})$}

For overcoming the issue obtained in Static Access Frequency Technique the DAFN (Dynamic Access Frequency and Neighbourhood) technique has been brought in. The identification and the access frequency at relocation period is obtained in the same technique by the mobile hosts. The replicas could be allocated in the equal method as in case of SAF technique. Then, if there can be duplicate allocation of a facts item among two neighbouring cell hosts, the cell host with the decrease get entry to frequency to the facts object adjustments the reproduction to a few different duplicates. [9]The node having excessive get admission to frequency will save its duplicate, if two mobile nodes have the identical statistics object. At every relocation duration, the cell nodes interchange statistics approximately duplicates allotted inside the reminiscence

When converting the reproduction, amongst facts things whose duplicates won't be allotted at one of the 2 hosts, a brand-new records object duplicated is chosen where the get entry to frequency cost to this object is the biggest some of the highest valuable things. It removes duplicate issuance amongst adjacent hosts.

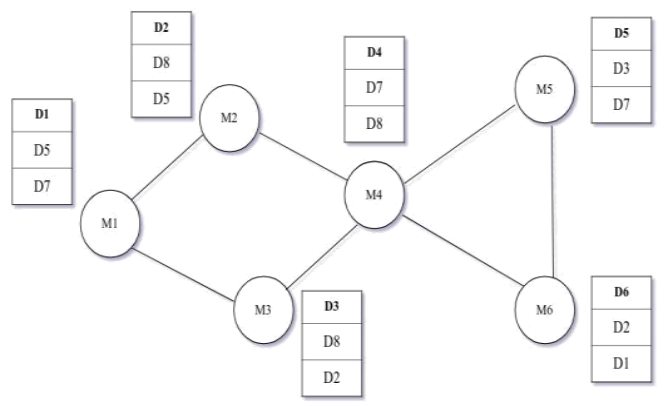

Fig 6.Node table DAFN Method.
Above Fig, Suggests DAFN approach in which duplicate issuance of memory, D8 considers area from M2 to M4, additionally the replication of D8 is between M3 and M4, and D7 is among M4 and M5.[10] The replica allocation cannot be accomplished at mobile hosts in addition to muddled connection, provided that the network topology alteration is depleted during the whole of the decapitation of this method.

\section{III.CHALLENGES IN EXISTING METHODS}

A considerable negative aspect in MANETs is that the prevailing existence of network bisection appropriate to the unfettered development of the mobile nodes central the network. This leads to a few statistics acquiring unreachable for few of the vertices. Thus, statistics approachability ought to be thought of rigorously in MANET. All mobile node in MANET needs the assistance of the substitution vertices to forward the packets. The vertices neighbourhood entity envisions to pause for a designated interruption between subsequent transmissions. Nevertheless, a mobile node may act because of network blockage and selfishness. Existing method encounters with several problems along with -

1. Poor throughput Less efficient

2. Higher packet drop rate

3. Lesser packet delivery rate

4. Poor selfish node detection Poor reproduction allocation

\section{IV.PROPOSED SYSTEM}

Replica allocation is important even though network problems are essential in a MANET, data services to the users is the surplus goal. For the benefit of other nodes, a selfish node might not proportion its own memory area to keep duplicate. [11]This can be erect standard peer-to-peer utility. A selfish node may not be sharing its intrinsic memory area for storing duplicate for the profit of remaining nodes. We can without difficulty discover these kind of issues in an average peer-to-peer utility.

It includes 3 components

1. Detect selfishness nodes Building SCF-tree

2. Replica Allocation

3. Detecting Selfish Nodes

Based on total credit score risk score, the selfish nodes can be detected. Credit risk rating can be explained as given below,

Credit Risk =anticipated threat $/$ anticipated price

The "degree of selfishness" of each node estimates for all of its related nodes primarily depending on the rating. The selfish node characteristics establishes which can pressure the selfish duplicate issuance hassle for deciding both predicted price as well as predicted hazard. Node precise and query processing-specific selfish characters. A particular node characteristic is a selfish node, The degree of selfishness can be explained as the size of shared memory 
space and/or the wide variety of shared statistics objects.

Algorithm for selfish node detection:

/* Ni selfishness node detection algorithm */

$\operatorname{detect}()\{$

for (connection $N_{k}$ )

if $\left(N_{i}\right.$ has allocated replica to $\left.N_{k}\right)$ is non-selfish

else $N_{k}$ is selfish; \}

wait;

if (connection $N_{k}$ ) \{

for $\left(N_{i}\right.$ has issued duplicate to $\left.N_{k}\right)\{$

$N D_{k}^{i}=$ allocation of duplicate;

$S S_{k}^{i}=$ Total size;

\{

$N D^{i}==0$;

$S S_{k}^{i}=$ Size;

\}) \}

\section{A.. Building SCF-Tree:}

The principal goal of novel duplicate issuance methods is to decrease site visitors overhead, to obtain the excessive facts approachability. Provided that very different duplicate issuance methods will issue with no communication with different vertices, then the visitors aerial reduces.[12] Contemplate the elementary SCF-tree topology $G$ that involves vertices along with connections, $N=(G, L)$. Here $G$ is variety of vertices where $L$ will be range of hyperlinks. The SCF-tree contains simplest non-selfish vertices, want to calculate the selfishness degree for the reproduction allocation within the MANET. The SCF-tree resides of only non-selfish nodes, the measurement of the degree of selfishness to apply real-world relationship management to duplicate issuance in a MANET should be done.

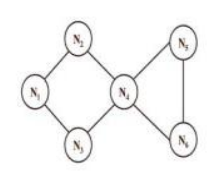

(a) Sample topology $G$

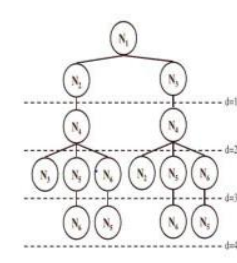

(b) SCF-tree of $N_{1}$

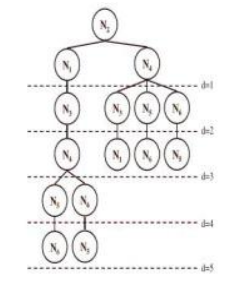

(c) SCF-tree of $\mathrm{N}_{2}$
Fig. 7Tree Diagram of SCF tree .

Every vertex will have a parameter d, the depth of SCF-tree. Initially $\mathrm{Ni}$ adds the vertices that are connected to $\mathrm{Ni}$ by hop to Ni's child nodes when Ni gets it's own tree. Thereby, Ni will check recursively the child nodes of the added vertices, until the depth of the SCF-tree is equal to t. Multiple routes may exist for some nodes from the root node in a SCF-tree. For example, N1 has two itineraries, N2 and N1 sets its own parameter. [13]As these several routes confer high stability, we issue more duplicates to the vertices that have several routes from the root node. All of the each node gets update to its particular tree based on the network topology. Below is the scf tree algorithm

00:/*Ni makes SCF-tree*/

build Scf-Tree()\{

add $N_{i}$ as root node;

Check smallnodes $\left(N_{i}\right)$;

return SCF-tree;

Check small nodes $\left(N_{j}\right)\{$

/*INa j are adjacent nodes to $\mathrm{Nj} * / 07$ :(each node $N_{a}$ belong $\left.\operatorname{to} N_{j}^{a}\right)\{$

If $\left(\mathrm{d}<\right.$ distance of $N_{a}$ and root $)$

continue;

else ( $N_{a}$ is an founder of $N_{j}$ in $T_{i}^{S C F}$ )

continue;

$\left\{\right.$ add $N_{a}$ to $T_{i}^{S C F}$ as a child of $N_{j}$;

Check small nodes $\left(N_{a}\right)$;

\}) \}

\section{B. Replica Allocation}

A vertex issues re-allocation at each relocation length. Every node will ask non-selfish vertices interior its SCF-tree to maintain reproduction on the identical time as it can not preserve duplicate in its neighbourhood memory place. As the SCF-tree based absolutely duplicate issuance is finished in a very allotted way, each vertex regulates duplicate allocation for my part with no connection with great vertices.[14] As each vertex will have its very non-public SCF-tree, it could execute duplicate issuance at its preference.

Replica is easily allocated at every node in decreasing order of its personal get entry to density. The one which is pretty unique is present group-primarily based replica allocation techniques in which duplicates are issued depending on the get means of approach to density of institution participants. A node regulates the concern for replica allocation. The precedence is primarily established on Breadth First Search (BFS) order. [15][16]The stippled one's represents the responsibility for replica allocations. After construction

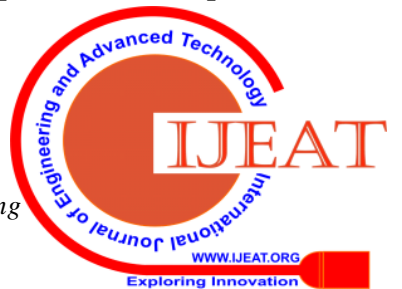


of SCF-Tree, replica allocation is completely done[17][18][19][20].

Algorithm for replica allocation:

/*Ni replica allocation */

allocation of replica( )

$K_{i}=\operatorname{priority}\left(T_{i}^{S C F}\right)$;

For (all data belongs to $\left.I D_{i}\right)\{$

If $\left(N_{s}=\mathrm{s}\right.$ is full;

issue duplicate of the information to $N_{s}$

else $\left\{/ * N_{s}\right.$ is not complete $* /$

issue duplicate of the information to the target node;

/* the marked node is chosen from $K_{i}^{* /}$

If $\left(M_{p}\right.$ is not full)

issue duplicate of the data to $\left.\left.M_{p} ;\right\}\right\}$

While (during relocation period) \{

( $N_{k}$ requests allocation of $C_{q}$ )

allocate others $\left.\left.\left(N_{k}, C_{q}\right) ;\right\}\right\}$

make $\operatorname{action}\left(T_{i}^{S C F}\right)\{$

for(top nodes in $\left.T_{i}^{S C F}\right)\{$

top node in $T_{i}^{S C F}$;

add the selected vertex id to $K_{i} ;$ \}

return $K_{i} ; ;$

duplicate issuance to others $\left(N_{k} ; D_{k} ;\right)\{$

if $\left(N_{k} ;\right.$ is in $T_{i}^{S C F}$ and $N_{i} ;$ ) won't hold $\left.\left.C_{q} ;\right)\right)\{$

$\operatorname{if}\left(M_{p} ;\right)$ is complete)don't issue $C_{q} ;$ )to $M_{p} ;$;

else $\{/ * \mathrm{Mp}$ is not complete*/

$\left(N_{i} ;\right)$ carries duplicate in $\left.\left.M_{p} ;\right)\right)$

replace $C_{q} ;$;

\{

/* $\mathrm{Nh}$ is the highest $n C R_{i}^{h}$ nodes which is done to replica allocation to $\mathrm{Mp}^{* /}$

if $>n C R_{i}^{h}$ ) replace requested replica by $N_{h}$ with $C_{q}$;

When $N i$ is not always a totally selfish node, $N i$ keeps its reminiscence space $M p$ for the appeal from available nodes, say $N_{k}$. Then, Ni have to arbitrate to agree the duplicate allocation request. If $N i$ 's $M p$ due, the duplicate of $C q$ can be given as Mp. If $N i$ 's $M p$ is complete and $N_{i}$ caarries any duplicate issued by means of itself in its $M_{p}, N_{i}$ replaces the reproduction n with $C q$.[17] If $N i$ won't keep any duplicate issued through itself in its $M p$ and $M_{p}$ is full, $N_{i}$ differentiates the $n C R_{i}^{h}$ with $n C R_{i}^{k}$, wherein $N_{h}$ is the node with the highest nCR rating the various nodes that allocated duplicate to $N_{i}$ 's Mp. If $n C R_{i}^{h}$ becomes more than $n C R_{i}^{h} N_{i}$ will replace the duplicate asked with the aid of $N_{h}$ with $C_{q}$.

\section{IV.CONCLUSION}

The trouble of selfishness nodes from the duplicate issuing thoughts-set is discussed and it is named as selfish duplicate issuance. This artwork become inspired through the reality that a selfish duplicate issuance needs to result in common awful information approachability in a MANET. A selfish node recognition approach and novel duplicate issuance strategies to cope with the selfish duplicate issuance successfully were presented. These presentes techniques have got the inspiration from the actual-global inspections in economics in phrases of credit score rating threat. MANET is a community with collection of movable nodes. Some of the nodes are selfish in nature. These selfish nodes are making a problem in replica allocation. A selfish node recognition technique and novel duplicate issuance methods for handling the selfish duplicate issuance properly were proposed. Data Accessibility, cost of the communication and query delay can be reduced by the proposed methodology. It also helps in the detection of time and maximizes the accuracy. The concept of credit risk from economics to recognize selfish nodes is applied. Each node in a MANET computes credit risk data on other connected nodes separately for measuring the degree of selfishness. Each node in a MANET computes credit threat facts on different related nodes in our view to degree of selfishness.

\section{REFERENCE}

[1]Sonali Karegaonkar and Archana Raut, "Improving Target Coverage and Network Connectivity of Mobile Sensor Networks", International Journal of Science and Research(IJSR), Vol.4, Issue No. 4, April 2015.

[2]D. Prasad, "Enhancing Target Coverage and Network Connectivity of Mobile Sensor Networks", International Journal of Innovative Research in Computer and Communication Engineering (IJIRCCE), Vol.4, Issue 1, January 2016.

[3] Mr. Mayur C. Akewar and Dr. Nileshsingh V. Thakur, "A study of Wireless Mobile Sensor Network deployment”, International Journal of Computer Networks \& Wireless Communication (IJCNWC), Vol.2, No. 4, August 2012.

[4] Arivubrakan P, Sarma Dhulipala V.R, 2012.Energy Consumption Heuristics In Wireless Sensor Networks. IEEE, International Conference on Computing, Communication and Applications (ICCCA), pp.1-5.

[5] Jae-Ho Choi, Kyu-Sun Shim, "Handling Selfishness in Replica Allocation over a Mobile Ad Hoc Network" SangKeun Lee, and Kun-Lung Wu, Fellow, IEEE.2012.

[6] T. Hara, "Effective Replica Allocation in Ad Hoc Networks for Improving Data Accessibility," Proc. IEEE INFOCOM, pp. 15681576, 2001. 
[7 ] T. Hara and S.K. Madria, "Data Replication for Improving Data Accessibility in Ad Hoc Networks," IEEE Trans. Mobile Computing, vol. 5, no. 11, pp. 1515-1532, Nov. 2006.

[8] Y. Liu and Y. Yang, "Reputation Propagation and Agreement in Mobile Ad-Hoc Networks," Proc. IEEE Wireless Comm. And Networking Conf., pp. 1510-1515.

[9] S. Marti, T. Giuli, K. Lai, and M. Baker, "Mitigating Routing Misbehavior in Mobile Ad hoc Networks," Proc. ACM MobiCom, pp. 255-265, 2000.

[10] L. Anderegg and S. Eidenbenz, "Ad Hoc-VCG: A Truthful and Cost-Efficient Routing Protocol for V. Srinivasan, P. Nuggehalli, C. Chiasserini, and R. Rao, "Cooperation in Wireless Ad Hoc Networks," Proc. IEEE INFOCOM, pp. 808-817, 2003.

[11] Hales, "From Selfish Nodes to Cooperative Networks - Emergent Link-Based Incentives in Peer-to-Peer Networks," Proc. IEEE Int'l Conf. Peer-to-Peer Computing, pp. 151-158, 2004.

[12] S.U. Khan and I. Ahmad, "A Pure Nash Equilibrium-Based Game Theoretical Method for Data Replication across Multiple Servers," IEEE Trans. Knowledge and Data Eng., vol. 21, no. 4, pp. 537-553, Apr. 2009.

[13] M.J. Osborne, An Introduction to Game Theory. Oxford Univ., 2003.

[14] B.-G. Chun, K. Chaudhuri, H. Wee, M. Barreno, C.H. Papadimitriou, and J. Kubiatowicz, "Selfish Caching in Distributed Systems: A Game-Theoretic Analysis," Proc.

[15] B.-G. Chun, K. Chaudhuri, H. Wee, M. Barreno, C.H. Papadimitriou, and J. Kubiatowicz, "Selfish Caching in Distributed Systems: A Game-Theoretic Analysis," Proc. ACM Symp. Principles of Distributed Computing.

[16] N. Laoutaris, G. Smaragdakis, A. Bestavros, I. Matta, and I.Stavrakakis, "Distributed Selfish Caching," IEEE Trans. Parallel and Distributed Systems, vol. 18, no. 10, pp. 1361-1376, Oct. 2007.

[17] Gao Jun Fan and ShiYao Jin, "Coverage Problem in Wireless Sensor Network: A Survey", Journal of Networks, Vol. 5, No. 9, September 2010.

[18] I. E. Korbi and S. Zeadally, "Energy-aware sensor node relocation in mobile sensor networks," Ad Hoc Network., vol. 16, no. 1, pp. $247-$ 265, 2014.

[19] I. F. Akyildiz, W. Su, Y. Sankarasubramaniam and ECayirci, "Wireless Sensor Networks: A Survey," in Elsevier Computer Networks, Volume: 38, Issue:2, Page(s):393-422, 2002.

[20] Raymond Mulligan and Habib M. Ammari, "Coverage in Wireless Sensor Networks: A Survey”, Macrothink Institute, ISSN 1943-3581, Vol.2, No.2, 2010.

\section{AUTHORS PROFILE}

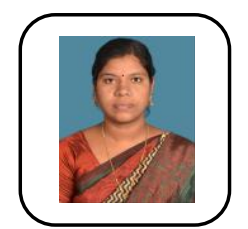

B.Umamaheswari,Asst.Professor, in the department of CSE,in veltech university having 4 years of teaching experience.I have completed M.E, Software engineering,Anna University. My Area of research is Mobile Adhoc networks.

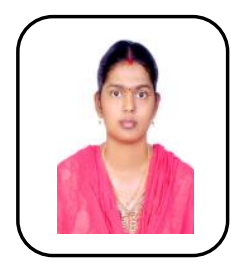

Ms.P.Arivubrakan,Asst.Professor, in the department of CSE, in veltech university, having 3 years of experience in the field of wireless sensor network, I have completed my M.E in pervasive computing technologies,My area of research in the sensor networks, IOT ,adhoc networks and pervasive computing.Reasearch articles are published in IEEE and Science Alert Journals. 